\title{
Umsetzung und Nutzenbewertung von Handlungsempfehlungen zur Patientensicherheit in der ambulanten operativen Versorgung
}

\section{Implementation and Benefit Evaluation of Recommendations for Patient Safety in Ambulatory Surgical Care}

Autoren

Institute

\section{Monaca ${ }^{1}$, M. Buchmann², T. Manser}

${ }^{1}$ Institut für Patientensicherheit, Universitätsklinikum Bonn, Bonn

${ }^{2}$ Kassenärztliche Vereinigung Westfalen-Lippe, Geschäftsbereich Versorgungsqualität, Dortmund
Schlüsselwörter

- Patientensicherheit

- Handlungsempfehlungen

- Ambulante Versorgung

- Niedergelassene Ärzte

- Unerwünschtes Ereignis

Key words

patient safety

practice recommendations

- ambulatory care

physicians in private practice

- adverse event

\section{Bibliografie}

DOI http://dx.doi.org/ 10.1055/s-0042-106896

Online-Publikation: 14.6.2016

Gesundheitswesen 2016;

78 (Suppl. 1): e103-e109

(c) Georg Thieme Verlag KG

Stuttgart · New York

ISSN 0949-7013

Korrespondenzadresse

\section{Clara Monaca, M.Sc. Public}

Health

Institut für Patientensicherheit Universitätsklinikum Bonn

Stiftsplatz 12

53111 Bonn

Clara.Monaca@ukb.uni-bonn.de

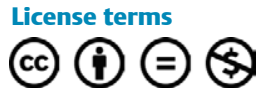

\section{Zusammenfassung}

$\nabla$

Ziel der Studie: Ziel der vorgestellten Evaluationsstudie war die Erfassung des Umsetzungsgrades von Handlungsempfehlungen zur Patientensicherheit in der ambulanten operativen Versorgung sowie die Nutzenbewertung seitens ambulant operierender Ärzte. Auf Basis von 2 Handlungsempfehlungen des Aktionsbündnisses Patientensicherheit wurden Handlungsempfehlungen zur Patientensicherheit von der Kassenärztlichen Vereinigung Westfalen-Lippe [KVWL] spezifisch für die ambulante operative Versorgung formuliert. Diese Empfehlungen wurden im Jahr 2013 an alle ambulant operierende Ärzte in Westfalen-Lippe versandt.

Methodik: Mittels eines schriftlichen Fragebogens wurden alle inhaltlichen Aspekte der beiden Handlungsempfehlungen erfragt. Weiter wurden der Umsetzungsgrad und Nutzen der empfohlenen Maßnahmen sowie eine subjektive Bewertung der Vorteile und Herausforderungen zur Implementierung erhoben. Der Fragebogen wurde Ende 2014 an 2454 ambulant operierende Ärzte verschickt. Der Befragungszeitraum betrug 7 Wochen. Im Rahmen der Datenanalyse wurden die quantitativen Ergebnisse deskriptiv aufbereitet sowie eine thematische Zusammenfassung der Freitextantworten zu den offenen Fragen vorgenommen.

Ergebnisse: Die Teilnahmerate betrug 17\% $(n=405)$. Die Handlungsempfehlungen waren 86\% der Befragungsteilnehmenden bekannt. Ein Großteil der empfohlenen Maßnahmen wurde in mehr als 50\% der teilnehmenden Einrichtungen systematisch umgesetzt. Als Vorteile der Handlungsempfehlungen wurde primär die interprofessionelle Sensibilisierung für das Thema Patientensicherheit genannt. Weiterer Bedarf an Informationen und Handlungsempfehlungen wurde $\mathrm{zu}$ folgenden Themengebieten angegeben: Risiko- und Fehlermanagement, Umsetzung

\section{Abstract \\ $\nabla$}

Objective: The aim of this study was to evaluate the degree of implementation of recommendations for patient safety in ambulatory surgical care and their benefit as perceived by surgeons in the ambulatory sector. Based on 2 practice recommendations issued by the Association of Statutory Health Insurance Physicians in Westphalia-Lippe, recommendations were formulated specifically for ambulatory surgery and distributed in 2013 to all physicians licensed to conduct ambulatory surgery in Westphalia-Lippe.

Methods: We conducted a written survey covering all safety measures addressed by the 2 practice recommendations and assessed the degree of implementation and the perceived benefit for each of these measures as well as the strengths of the recommendations and the challenges of implementing them. The survey was distributed in late 2014 to 2454 surgeons in the ambulatory setting. The survey period was 7 weeks. The analysis of the quantitative data was mainly descriptive and we conducted thematic summaries of free text answers to open-ended questions. Results: The participation rate was $17 \%$ $(n=405)$. The recommendations were known to $86 \%$ of the respondents. The majority of recommended safety measures had been implemented systemically in more than $50 \%$ of the participating institutions. An increased interprofessional awareness of patient safety measurements was reported as the main impact of the recommendations. Respondents indicated further need for information and practice recommendations concerning the following topics: risk and error management, implementation of the Medical Devices Act, hygiene in medical practice and processing of instruments.

Conclusion: This study highlights the valuable contribution practice recommendations can make to patient safety improvement in ambulatory sur- 
des Medizinproduktegesetzes, Hygiene in der Praxis sowie Aufbereitung von Instrumenten.

Schlussfolgerung: Diese Studie weist darauf hin, dass praxisorientierte Handlungsempfehlungen einen wertvollen Beitrag zur Förderung der Patientensicherheit in der ambulanten operativen Versorgung leisten können. Die Ausweitung der Handlungsempfehlungen auf weitere Regionen sowie, nach entsprechender Anpassung, auch auf andere Bereiche der ambulanten Versorgung ist daher zu empfehlen.

\section{Einleitung}

Die World Health Organization veröffentlichte im Jahr 2008 mit der zweiten globalen Forderung zur Verbesserung der Patientensicherheit in der stationären Versorgung das Manual „Safe Surgery - Saves Lives“ [1]. Die hierin publizierten Checklisten zur Umsetzung von Maßnahmen zur Patientensicherheit in der Chirurgie (z. B. richtiger Patient, richtiger Operationsraum, richtige Seite) konnte die Komplikationsrate und Mortalität deutlich senken [2]. Wie dieses Beispiel zeigt, liegt der Fokus von systematischen Maßnahmen für eine sichere Patientenversorgung vielfach auf der stationären Krankenhausversorgung, während nur wenige Studien und konkrete Empfehlungen zur Patientensicherheit im ambulanten operativen Sektor vorliegen [3]. Die hier vorgestellte Studie evaluiert einen innovativen Ansatz, um diese Lücke zu schließen.

Zur Förderung von spezifischen Patientensicherheitsmaßnahmen im ambulanten operativen Bereich wurden 20132 Handlungsempfehlungen des Aktionsbündnisses Patientensicherheit - Vermeidung von Eingriffsverwechselungen in der Chirurgie sowie zur sicheren Patientenidentifikation [4,5] -zielgruppenspezifisch für Praxisteams im ambulant operativen Bereich formuliert. Handlungsempfehlungen eignen sich besonders für sicherheitskritische Situationen als praxistaugliche Ratgeber und Anleitungen, die idealtypische Abläufe abbilden. Für die Patientensicherheit fokussieren diese gemäß dem Aktionsbündnis Patientensicherheit „Strategien zur Vermeidung von Risiken und Fehlern, die für die Patientin oder den Patienten schädliche Folgen haben könnten“ [6]. Zur Vermeidung von Eingriffsverwechselungen beim ambulanten Operieren wurde ein Vier-Stufen-Handlungs-Plan beschrieben, um das Risiko einer Eingriffsverwechselung zu minimieren: Identifikation und Aufklärung, Markierung des Eingriffsortes, Identifikation des richtigen Patienten für den richtigen Operations-/Eingriffsraum und Team-Time-Out im Operations-/Eingriffsraum [7]. Die Handlungsempfehlung zur sicheren Patientenidentifikation enthält 6 Aspekte zur sicheren Patientenidentifikation: Zuordnung von Befunden nach Abgleich von Identifizierungsmerkmalen, Akzeptanz der Überweisungsscheine nur mit Stammdatensatz und vollständigem Absender, digitalisierte Erfassung des Stammdatensatzes, Ermittlung von Identifizierungsmerkmalen vor jeder diagnostischen und therapeutischen Maßnahme, Kennzeichnung und Abgleich von Proben sowie Anlegen eines Patientenarmbandes [8].

Im Juli 2013 wurden die Handlungsempfehlungen an alle Ärzte mit einer Genehmigung zum ambulanten Operieren in Westfalen-Lippe verschickt. Die Ärzte sollten die Relevanz der Empfehlungen für ihren Praxisalltag erkennen, Anregungen übernehmen und bestenfalls in das lokale Qualitäts- und Risikomanagementsystem integrieren. Im Vordergrund der Ende 2014 durch das Institut für Patientensicherheit der Universitätsklinik Bonn durchgeführten Evaluation der Empfehlungen standen folgende gical care. Their dissemination to other regions as well as to other ambulatory care settings such as family practice can therefore be recommended.

Forschungsfragen: Wie ist der Umsetzungsgrad der beiden Handlungsempfehlungen in der Praxis? Welchen Nutzen können die Praxen aus den Handlungsempfehlungen ableiten? Welche Vorteile und Herausforderungen werden zur Implementierung berichtet? Was für praxisrelevante Handlungsempfehlungen werden zukünftig benötigt?

\section{Methodik}

$\nabla$

Die Grundlage der Evaluationsstudie bildete ein Fragebogen, der Ende 2014 an alle zu diesem Zeitpunkt zugelassenen ambulant operierenden Ärzte in Westfalen-Lippe verschickt wurde $(n=2454)$. Die Befragung erfolgte ohne materielle Anreize bzw. wurden keine sonstigen Incentives bereitgestellt. Die Teilnahme war freiwillig und anonym, sowohl postalisch als auch online möglich und der Befragungszeitraum betrug 7 Wochen zwischen November und Dezember 2014. Eine Teilnahmeerinnerung erschien nach der Hälfte des Befragungszeitraumes in der Mitgliederzeitschrift der KVWL (KVWL Kompakt).

Der Fragebogen deckte alle inhaltlichen Aspekte der beiden Handlungsempfehlungen ab und ließ sich folgenden Themenbereichen zuordnen: Kenntnisnahme der Handlungsempfehlung, bisherige und aktuelle Vorgehensweisen zur Patientensicherheit, Umsetzungsgrad und Verwendbarkeit der Handlungsempfehlungen im Praxisalltag, Angaben zum Qualitätsmanagement in der Praxis, persönliche und demografische Daten. Des Weiteren wurden zur Bewertung des Nutzens 7 Aspekte befragt: Kostenintensität, Patientensicherheit, Praxisrelevanz, Praktikabilität, Umfang, Umsetzbarkeit und Zeitintensität. Die insgesamt 29 Fragen gliederten sich in 19 Items mit vorgegebenen Antwortmöglichkeiten und 10 offenen Fragen.

Um den Umsetzungsgrad der in den Handlungsempfehlungen beschriebenen Maßnahmen ableiten zu können, wurde zur Skalierung der Antworten das Transtheoretische Modell der Organisationsveränderung genutzt $[9,10]$. Anhand der 5 Stufen des Modells (systematisch umgesetzt; unsystematisch umgesetzt; umsetzung in den nächsten 12 Monaten geplant; diskutiert, bisher keine Umsetzungspläne; noch nicht damit befasst) ließ sich zum einen die Veränderungsbereitschaft untersuchen und zum anderen, inwieweit die Praxen bzw. befragten Personen bereits Veränderungen umgesetzt hatten. Die Anwendung des Transtheoretischen Modells zur Untersuchung von Implementierungsgrad und Veränderungsbereitschaft wurde bereits in Befragungen zum klinischen Risikomanagement erfolgreich eingesetzt [11].

Die Grundlage der Datenanalyse bilden eine deskriptive Auswertung der Antworten zu den einzelnen Items sowie eine thematische Zusammenfassung der offenen Fragen. Dabei wurden bei Auswahlfragen die gültigen Prozente gewertet. 


\section{Ergebnisse}

\section{Struktur der Teilnehmenden}

An der Befragung nahmen insgesamt 405 ambulant operierende Ärzte und sonstige Angestellte (z.B. Medizinische Fachangestellte, Qualitätsmanagementbeauftragte) teil. Dies entspricht einer Teilnahmerate von 17\%. 95 Personen arbeiten in Berufsausübungsgemeinschaften; davon gaben 40 Befragte ihre eigene Meinung und Einschätzung wieder und 55 füllten den Fragebogen gleichzeitig für Praxiskollegen aus, wodurch insgesamt 19\% der Grundgesamtheit durch die Antworten repräsentiert ist. Da insbesondere die demografischen Angaben jedoch nicht dupliziert werden sollten, wurden diese nicht in die weitere Berechnung aufgenommen.

Nach Altersgruppen betrachtet, besitzen 75\% der Teilnehmenden bereits über 20 Jahre Berufserfahrung und $73 \%$ sind über 50 Jahre alt. 71\% der Praxen sind den Fachrichtungen Chirurgie, Gynäkologie, Ophthalmologie, Dermatologie sowie Hals-NasenOhren-Heilkunde zuzuordnen. Die fachärztliche Zuordnung der Befragten ist repräsentativ für die Verteilung der ärztlichen Fachrichtungen der in der Grundgesamtheit.

Systematische Vorgehensweisen zur Förderung der Patientensicherheit waren im Vorfeld in $91 \%$ der Praxen etabliert. Über 50\% gaben an, Praxisbesprechungen, klare Aufgabenverteilungen, Checklisten und Fortbildungen einzusetzen (siehe $\bullet$ Abb. 1 für detaillierte Ergebnisse). Des Weiteren setzen 88\% der Praxen ein Qualitätsmanagement-System um und sind dementsprechend zertifiziert.

\section{Umsetzungsgrad der Handlungsempfehlungen}

Mehr als 86\% der Befragten waren die beiden Handlungsempfehlungen zur Patientensicherheit zum Zeitpunkt der Befragung bekannt und $65 \%$ der Teilnehmenden würden diese voll bzw. überwiegend weiterempfehlen. Diese hohe Weiterempfehlungsrate liefert bedeutende Hinweise für die Gesamtbewertung der Handlungsempfehlungen.

Nachfolgend werden zunächst die Ergebnisse zum Umsetzungsgrad der Handlungsempfehlung zur Vermeidung von Eingriffsverwechselungen dargestellt, dann die Ergebnisse zur sicheren Patientenidentifikation.

Die Ergebnisse zu der vierschrittigen Handlungsempfehlung zur Vermeidung von Eingriffsverwechselungen zeigen, dass alle Schritte in mehr als 60\% der teilnehmenden Praxen bereits systematisch bzw. unsystematisch umgesetzt wurden. Die differenzierten Ergebnisse sind in $\bullet$ Abb. 2 dargestellt.

Falls die empfohlenen Maßnahmen nicht umgesetzt wurden, wurde neben der besseren Praktikabilität der etablierten Methoden als Grund vorrangig angeführt, dass die Patienten persönlich bekannt seien. Zudem seien die Maßnahmen nach Einschätzung der Befragten bei Eingriffen unter Lokalanästhesie,

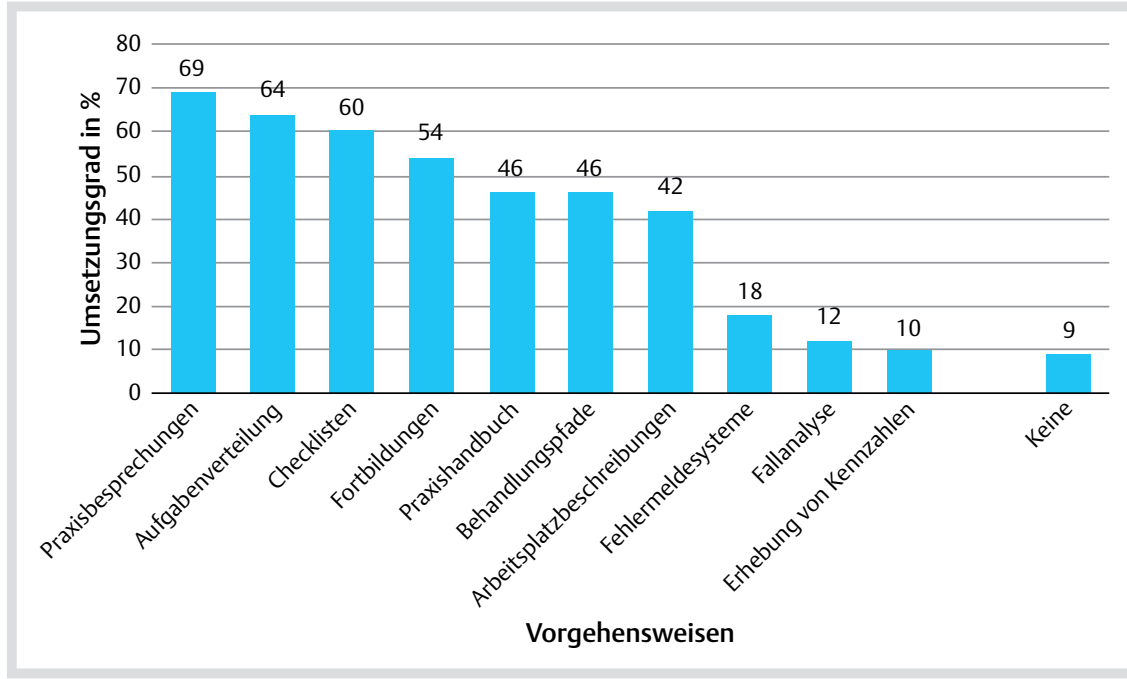

Abb. 1 Umsetzungsgrad von systematischen Vorgehensweisen zur Förderung der Patientensicherheit (Auswahlfrage; Gültige Prozente der Einzelitems)

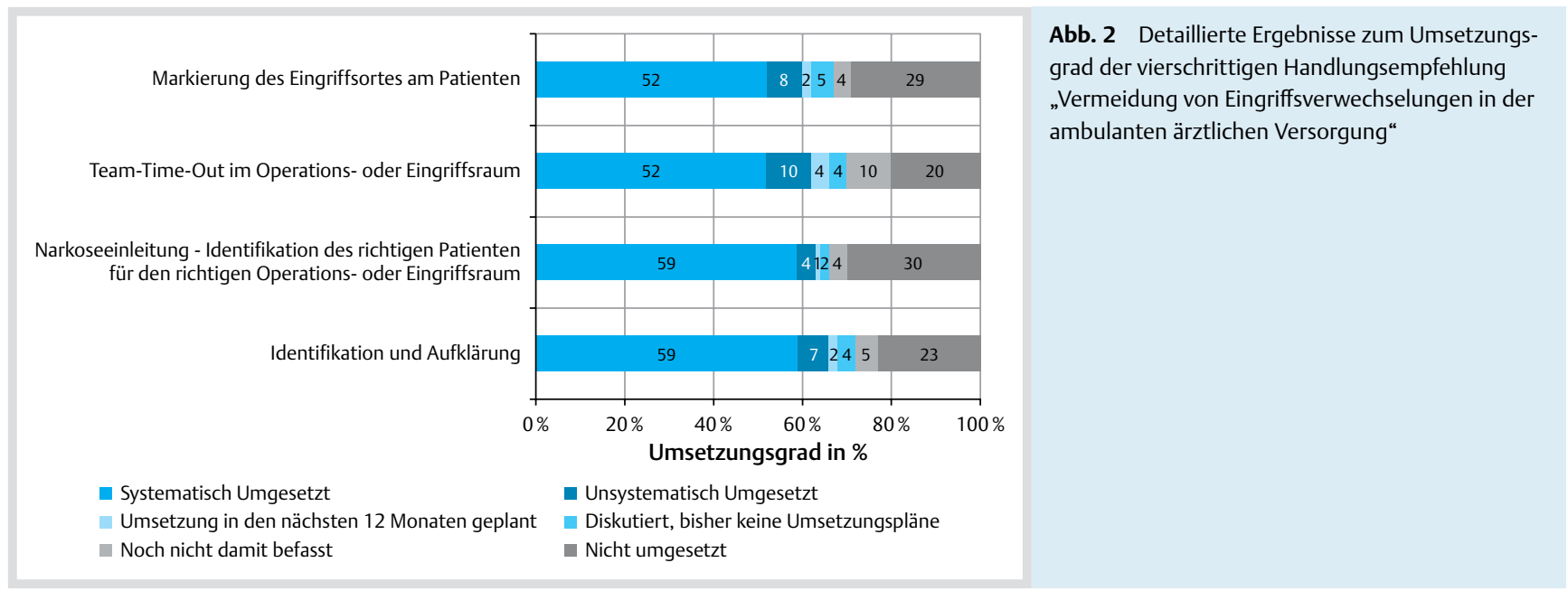




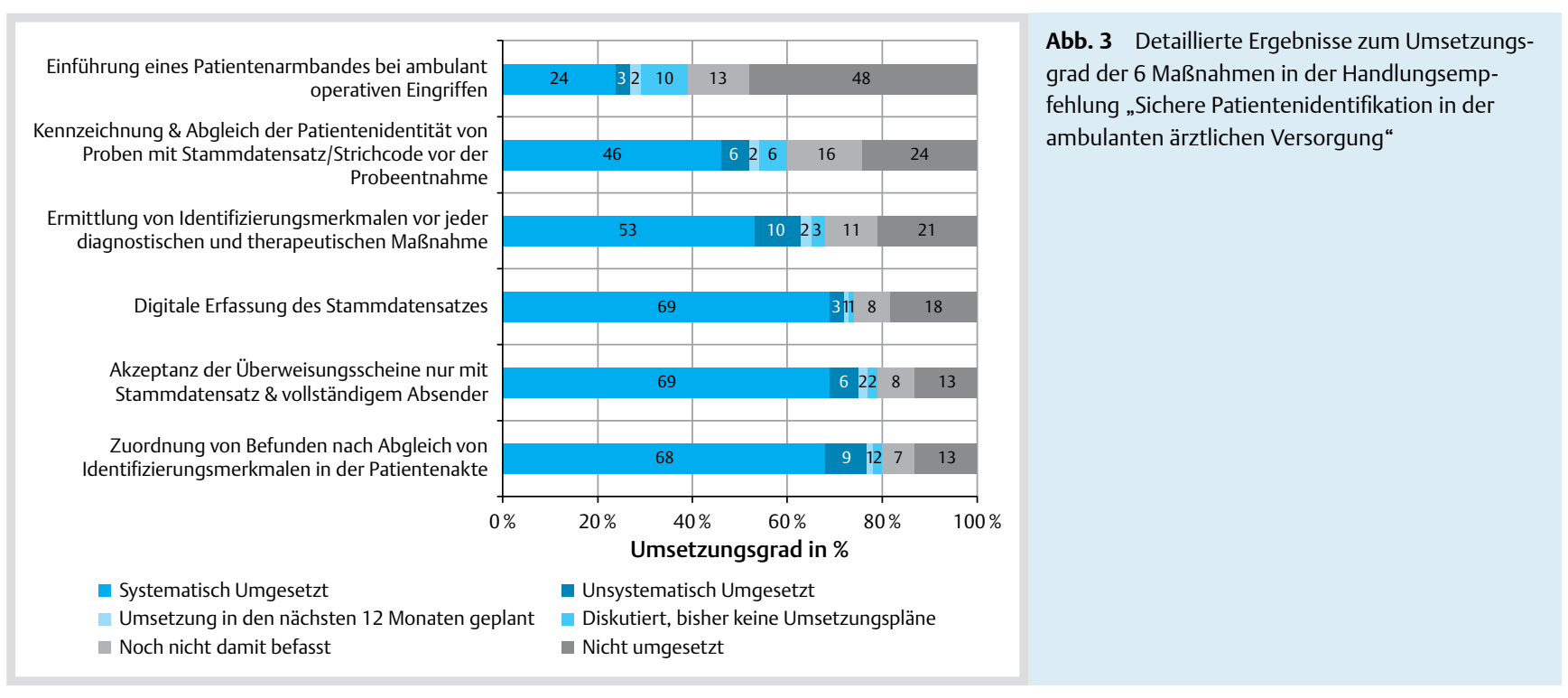

bzw. wenn keine Narkose durchgeführt wird, irrelevant und bei den vorgenommenen Eingriffen im Bereich Gynäkologie, Urologie, Hals-Nasen-Ohren Heilkunde oder Ophthalmologie nicht möglich bzw. nicht erforderlich. Als weitere Gründe für Nichtumsetzung wurde angeführt, dass nur ein einziger Operationsraum vorhanden sei oder dass die Verantwortlichkeit zur Nutzung entsprechender Checklisten der Anästhesieabteilung obliege. Die am häufigsten genannte alternative Vorgehensweise war das Führen von persönlichen Gesprächen mit dem Patienten vor der Operation.

Die Ergebnisse zu den 6 empfohlenen Maßnahmen zur sicheren Patientenidentifikation zeigen, dass 5 Maßnahmen in mehr als $50 \%$ der Praxen bereits systematisch bzw. unsystematisch umgesetzt wurden (siehe $\bullet$ Abb. 3 für detaillierte Ergebnisse).

Zwischen 77 und $72 \%$ gaben an, die empfohlenen Maßnahmen zur korrekten Zuordnung von Befunden, die Akzeptanz von Überweisungsscheinen mit dem Stammdatensatz und einem vollständigen Absender sowie die digitale Erfassung des Stammdatensatzes umgesetzt zu haben. Aufgrund des hohen Umsetzungsgrads wurden kaum Angaben zu alternativen Maßnahmen gemacht. Einige Praxen gaben jedoch an, es gebe im Praxisalltag keine Überweisungen und der Ausdruck von der elektronischen Patientenakte werde als Stammdatensatz genutzt.

Der Umsetzungsgrad der 3 weiteren Maßnahmen lag zwischen 63 und 27\%. Als alternative Maßnahme zur Empfehlung zur Ermittlung von Identifizierungsmerkmalen vor jeder diagnostischen und therapeutischen Maßnahme wurden u.a. Patientengespräche vor der Operation genannt. Falls keine Kennzeichnung von Proben zum Abgleich der Patientenidentität mit dem Stammdatensatz/Strichcode vor der Probeentnahme umgesetzt wurde, wurde dieses damit begründet, dass in der Praxis keine Probeentnahme erfolge oder die Patienten persönlich bekannt seien. Auch hinsichtlich alternativer Maßnahmen zur Verwendung eines Patientenarmbandes wurde angeführt, dass die Patienten persönlich bekannt seien, die Operationen nur unter Lokalanästhesie stattfänden und/oder kein hohes Patientenaufkommen vorliege. Die Befragten gaben als häufigste alternative Vorgehensweise die Patientenidentifikation durch persönliche Gespräche an.

\section{Vorteile, Herausforderungen und Reaktionen auf die Handlungsempfehlungen}

Die Ergebnisse zu den Vorteilen, Herausforderungen und Reaktionen in der Praxis nach Erhalt der Handlungsempfehlungen basieren auf Freitextantworten auf offene Fragen. Diese Möglichkeit wurde von den Teilnehmenden rege genutzt. Wörtliche Zitate aus der Umfrage sind in kursiv abgedruckt.

\section{Vorteile der Handlungsempfehlungen}

Als Vorteile der Handlungsempfehlungen wurden primär die Erhöhung und Verbesserung der Patientensicherheit für alle Beteiligten (Patient, Arzt, Pflegekraft) genannt. In den Praxen habe eine Sensibilisierung für das Thema Patientensicherheit und insbesondere zur Vermeidung und Verringerung von Fehlern stattgefunden. Die Standardisierung von Arbeitsabläufen entsprechend der empfohlenen Maßnahmen sei für Praxen ein zusätzlicher Qualitätsgewinn sowie eine Zeitersparnis durch die als sinnvoll und praktikabel angesehene Systematik. Gleichzeitig würden die Empfehlungen als Grundlage für die Einarbeitung neuer Mitarbeiter genutzt. Eine mögliche Absicherung bei Haftpflichtfragen, eine Senkung von Haftpflichtansprüchen, Klagen und der Schutz vor Regress wurden von einigen Teilnehmenden als weitere Vorteile angegeben:

- „Neue Sichtweise! Regt zu Diskussionen an.“

- „Gefühl der Zufriedenheit durch den betriebenen Aufwand.“

Einige Ärzte sahen in den Handlungsempfehlungen keine Vorteile:

- „Keine wesentlichen, da zeitintensiv und zu bürokratisch (Zettelwirtschaft).“

- „Nichts, davor war ohne Bürokratie immer alles richtig.“

- „Sehr relevant für große Praxen mit hohem Operationsaufkommen und Kliniken. Für kleine Praxen eher irrelevant.“

Zudem seien die Patienten persönlich bekannt und Operationen würden nur unter Lokalanästhesie durchgeführt. Des Weiteren wurde beschrieben, dass Fehler bisher nicht vorgekommen seien oder äquivalente Maßnahmen im Vorfeld bereits etabliert waren.

\section{Herausforderungen bei der Umsetzung der Handlungsempfehlungen}

Die wahrgenommenen Herausforderungen zur Umsetzung der Handlungsempfehlungen lagen vor allem in einem erhöhten Zeitaufwand für die Implementierung sowie für den kontinuier- 
lichen Betrieb. Die Anpassung bzw. Umstellung des Managements und der Arbeitsanweisungen an ein neues System sowie der Dokumentationsaufwand wurden neben erhöhten Investitionskosten genannt. Die konsequente Beibehaltung des neu etablierten Systems sowie die Schulung des gesamten Teams wurden als weitere Herausforderungen aufgezeigt:

- "Sehr gut, aber sehr zeit- und kostenintensiv.“

- „Die Empfehlungen ersetzen nicht den Arzt-Patienten-Kontakt vor der Operation in meiner Praxis.“

In anderen Praxen seien hingegen keine Herausforderungen zu erkennen gewesen, da diese Prozesse bereits Routine seien bzw. die Umsetzung keine großen Hindernisse und keinen nennenswerten Aufwand dargestellt habe (siehe dazu auch die im Vorfeld etablierten Maßnahmen in $\bullet$ Abb. 1).

\section{Ausgelöste Reaktionen in den Praxen nach Erhalt der Handlungsempfehlungen}

Nach Erhalt der Handlungsempfehlung wurden ein erhöhtes Problembewusstsein und mehr Aufmerksamkeit für Fragen der Patientensicherheit wahrgenommen:

- „Unsere bisherigen Maßnahmen nochmal zu überprüfen und mehr Aufmerksamkeit für das Problem von mir und meinen Mitarbeitern.“

Teambesprechungen, das Critical Incident Reporting System (CIRS) und/oder ein Qualitätsmanagementsystem sowie einzelne Maßnahmen der Handlungsempfehlungen, die im bisherigen Praxisablauf gefehlt hätten, seien etabliert worden. Für den Praxisalltag bedeutete dies, dass Aufgaben neu verteilt, bisherige Arbeitsabläufe überprüft, überdacht und geändert wurden. Dadurch kam es auch zu mehr Dokumentationsaufwand. Positiv wurde angemerkt, dass die aktive Kommunikation im Team und mit den Patienten durch die Umsetzung der Empfehlungen gesteigert worden sei:

- „Pflicht der Lektüre durch alle Mitarbeiter und Aufnahme in das Qualitätsmanagement-Handbuch.“

- „Kooperation und Kommunikation im Team steigern und stärken.“ Bei Praxen, die die Inhalte der Maßnahmen bereits umgesetzt hatten, haben die Handlungsempfehlungen zumeist keine Reaktion ausgelöst. Andere Praxen gaben jedoch an, dass das etab- lierte System überprüft und diskutiert worden sei und die Umsetzung der Handlungsempfehlungen anschließend abgelehnt oder befürwortet wurde.

\section{Bewertung von Nutzen-Aspekten}

Wie aus $\bullet$ Abb. 4 ersichtlich, gaben die Teilnehmenden an, besonders zufrieden mit dem Aspekt der Patientensicherheit zu sein. Dies wird für die Handlungsempfehlung „Eingriffsverwechselungen“ etwas positiver bewertet (80 zu 75\%). Weiterhin zeigt sich, dass trotz des geäußerten Aufwands zur Umsetzung der Handlungsempfehlungen ein Großteil der Befragten (66 bzw. $60 \%$ mit der Umsetzbarkeit sehr zufrieden bzw. zufrieden ist. Obschon in den offenen Fragen die Praktikabilität als besondere Herausforderung gesehen wurde, zeigt sich in der Nutzenbewertung, dass zwischen 66 und $62 \%$ der Teilnehmenden hiermit zufrieden sind. Zudem ergaben sich für Praxisrelevanz (71 bzw. 69\%), Umfang (61 bzw. 54\%) und Zeitintensität (55 bzw. 50\%) hohe Zufriedenheitswerte. Die Zeitintensität ist der Aspekt, welcher die Befragten am ehesten unzufrieden bzw. sehr unzufrieden stimmte (26 bzw. 28\%). Der Aspekt der Kostenintensität zeigt den höchsten Anteil bei der Antwortskala „nicht beurteilbar" und die geringsten, aber noch immer vergleichsweise hohen Zufriedenheitsraten (52 bzw. 53\%).

\section{Weitere Anmerkungen für zukünftige \\ Handlungsempfehlungen}

Einige Teilnehmende gaben an, weitere Handlungsempfehlungen zu den Themen Hygiene, professionelles Praxis-Management und Gesundheitsförderung für Mitarbeiter zu wünschen. Zudem bestehe Bedarf an Informationen zu rechtlichen Fragen, Erfahrungsberichten von Kollegen, die über kritische Ereignisse bzw. Fehler berichten, sowie zu unterschiedlichen medizinischen Aspekten wie Onkologie oder chronische Wunden. Optimierungsvorschläge für zukünftige Handlungsempfehlungen waren z.B. der Wunsch nach mehr Partizipation bei der Entwicklung der Handlungsempfehlungen sowie Schulungen zu den Themen der Handlungsempfehlungen, die kostenfrei sein sollten und auch für nicht-ärztliche Mitarbeiter zugänglich sein sollten.

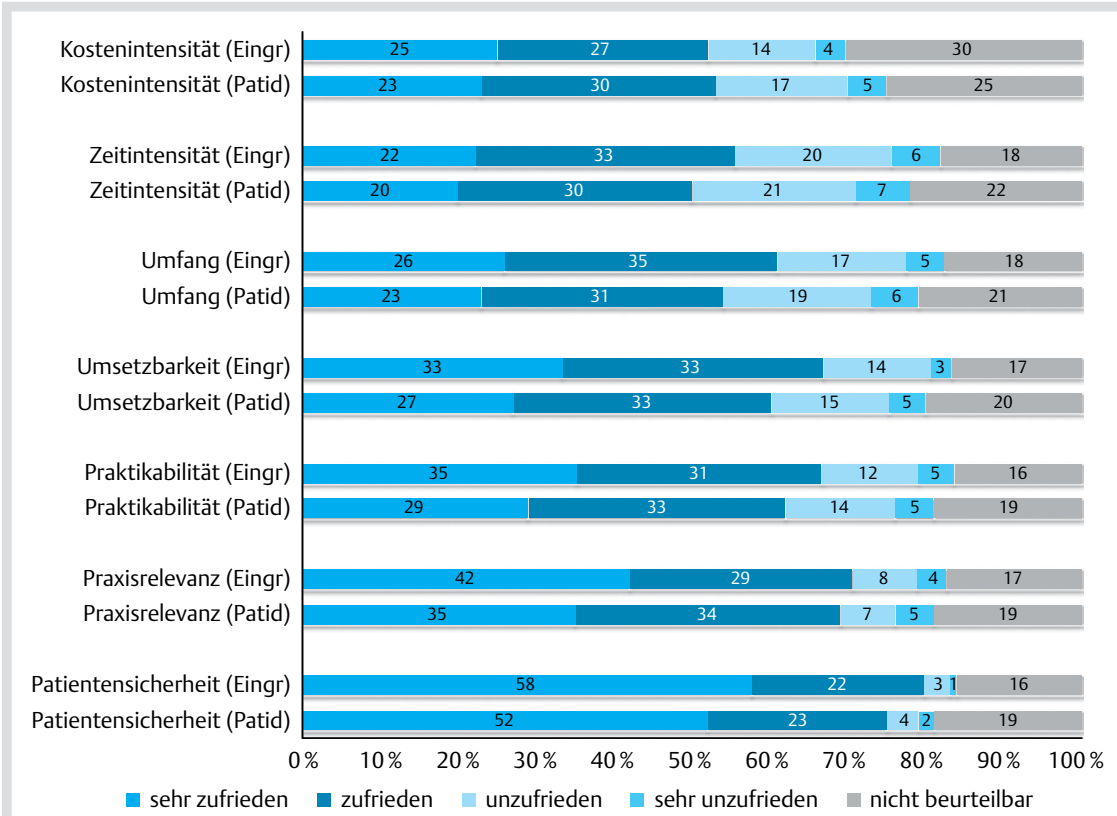

Abb. 4 Nutzenbewertung der beiden Handlungsempfehlungen.

Eingr: Handlungsempfehlung. Vermeidung von Eingriffsverwechslungen in der ambulanten Versorgung. Patid: Handlungsempfehlung. Sichere Patientenidentifikation in der ambulanten ärztlichen Versorgung. 


\section{Diskussion}

$\nabla$

In einer kürzlich veröffentlichten Studie erachteten $88 \%$ der befragten Chirurgen Patientensicherheit als sehr wichtig und 12\% als wichtig [12]. Die Umsetzung eines systematischen Vorgehens zur Erhöhung der Patientensicherheit bedarf jedoch der gezielten Unterstützung [13]. Als solche sind die hier untersuchten Handlungsempfehlungen zur Vermeidung von Eingriffsverwechselungen beim ambulanten Operieren und zur sicheren Patientenidentifikation zu verstehen.

Die hier vorgestellte Evaluation verdeutlicht, dass Handlungsempfehlungen einen praxisrelevanten und unterstützenden Beitrag zur Umsetzung von Maßnahmen zur Erhöhung der Patientensicherheit in der ambulanten Versorgung leisten können. Die beiden Handlungsempfehlungen haben nach Aussagen einiger Befragter zu einer Sensibilisierung für Fragen der Patientensicherheit beigetragen und wurden als praktikable Hilfestellung gesehen, um ein systematisches Vorgehen zu etablieren oder das bereits bestehende System zu optimieren.

Insgesamt ließ sich feststellen, dass die Teilnehmer der Befragung mit Ihren Praxisteams auf dem Weg sind, sich systematisch mit dem Thema Patientensicherheit zu befassen. Hierzu gehört es, abzuwägen, welche Aspekte der Handlungsempfehlungen für die tägliche Praxis relevant sind und welche spezifisch an den Praxisalltag angepasst werden müssen. Die Mehrzahl der Befragten beurteilte beide Handlungsempfehlungen als anwendbar. Die empfohlenen Maßnahmen wurden mehrheitlich umgesetzt und wirken sich nach Aussage der Teilnehmer im Praxisalltag positiv aus. Dies trifft insbesondere auf solche Praxen zu, die die Maßnahmen der Empfehlungen im Arbeitsalltag neu implementierten. Bei Praxen, die die Maßnahmen bereits vorher umgesetzt hatten, kam es zu wenigen oder keinen neuen Erkenntnissen. Daraus kann der Schluss gezogen werden, dass der Nutzen einer Handlungsempfehlung umso geringer ist, je höher der Entwicklungsstand in den Praxen bereits ist. Aber auch hier wurde berichtet, dass die Empfehlungen zur Reflexion bzw. Bestätigung des eigenen Vorgehens genutzt wurden. Das etablierte System kann anhand einer Handlungsempfehlung hinterfragt und durch die Übernahme einzelner Elemente angereichert werden. Die Studie zeigt, dass dies im Team mit ärztlichem und nicht-ärztlichem Personal diskutiert wurde.

Als problematisch ist jedoch ein Teil der angegebenen Begründungen anzusehen, warum bestimmte Maßnahmen nicht umgesetzt werden. Die Ergebnisse der Studie zeigen, dass einige Ärzte sich durch die Handlungsempfehlung in ihrer Individualität und Gestaltung des Praxisablaufs diszipliniert fühlen. In vielen Praxen gibt es gegen Handlungsempfehlungen und Leitlinien weiterhin große Vorbehalte. Aus der Literatur ist bekannt, dass Ärzte fürchten, an diesen im Falle einer rechtlichen Auseinandersetzung gemessen zu werden [14]. Oft haben die teilnehmenden Ärzte erwähnt, die Patienten persönlich zu kennen und führten ihre bisherige Null-Fehler-Quote auf eine gefestigte Arzt-Patienten-Beziehung zurück. Die aufgeführten Gründe reduzieren zwar als alternative Maßnahmen das Risiko, einen Patienten zu verwechseln, doch helfen sie nicht, das Risiko systematisch auszuschließen. Eine bewusste Kontrolle zur Patientenidentifikation im Rahmen eines mehrschrittigen systematischen Vorgehens bleibt darüber hinaus unerlässlich.

Die Befragungsergebnisse liefern auch Ansatzpunkte für eine weitere Erhöhung der Akzeptanz bzw. Umsetzung. So zeigte sich bspw., dass die im stationären Bereich weit verbreitete Terminologie aus den Empfehlungen der World Health Organization
„Safe Surgery - Saves Lives“ in der ambulanten Versorgung vielfach nicht bekannt ist. Die Verwendung des Begriffs „TeamTime-Out", welcher in den Handlungsempfehlungen nicht weiter erläutert wird, führte bei den Befragten teilweise zu Verunsicherung und auch zum Nicht-Beachten der Maßnahme. Daher werden zusätzliche Erläuterungen und Schulungen benötigt, um einen durchlässigeren Übergang von Empfehlungen vom stationären in den ambulanten Bereich zu gewährleisten. Zudem hoben die Befragten hervor, dass bereits bei der Entwicklung von Handlungsempfehlungen eine ausreichende Partizipation der Zielgruppe sichergestellt sein muss [15].

Bei der Interpretation der Ergebnisse dieser Studie ist zu beachten, dass nur innerhalb einer Region in Deutschland untersucht wurde. Aufgrund des heterogenen Gebiets der KVWL (städtische und ländliche Strukturen) sind die Ergebnisse jedoch durchaus auch für weitere Regionen repräsentativ. Darüber hinaus ist festzuhalten, dass die Teilnahmerate mit 17\% für eine Studie im Gesundheitswesen ohne Incentives in einem üblichen Bereich liegt (insbesondere für die ambulante Versorgung). Dennoch sind bei einer Teilnahmerate unter 70\% generell Selektionseffekte anzunehmen und eine Generalisierung der Aussagen zum Umsetzungsgrad für die Grundgesamtheit wäre unzulässig. Da die Struktur der teilnehmenden Ärzte bzw. Praxen durchaus der Verteilung in der Grundgesamtheit entspricht und damit für die Repräsentativität der Stichprobe spricht, kann von einer grundsätzlichen Umsetzbarkeit der Handlungsempfehlungen ausgegangen werden. Weiter wurden im Rahmen dieser anonymen Befragung, insbesondere in den qualitativen Aussagen zu den Handlungsempfehlungen, durchaus kritische Meinungen geäuBert, weshalb Antworttendenzen in Richtung sozialer Erwünschtheit als eher gering einzuschätzen sind.

In der Zusammenschau der Ergebnisse bleibt festzuhalten, dass praxisorientierte Handlungsempfehlungen für eine Vielzahl von Herausforderungen in der Patientenversorgung Hilfestellung bieten können. Strukturelle Veränderungen in der Gesundheitsversorgung führen dazu, dass sich die bisherige Situation niedergelassener Ärzte verändert. Das Bild einer langjährigen, persönlichen Arzt-Patienten-Beziehung wird immer weiter verzerrt. Die Anzahl der Einzelpraxen sinkt, Praxen schließen sich zusammen und betreuen immer mehr Personen. Des Weiteren steigt die Komplexität der Behandlungsmöglichkeiten auch im niedergelassenen Bereich [16]. Entsprechend steigen die Herausforderungen im Zusammenhang mit der Gewährleistung der Patientensicherheit. Es ist daher zu fordern, dass entsprechende systematische Maßnahmen für eine sichere Patientenversorgung flächendeckend umgesetzt werden, hierbei können Handlungsempfehlungen Unterstützung bieten. Die vorliegende Studie zeigt: es besteht durchaus weiterer Verbreitungsbedarf und eine Ausweitung auf weitere Themen, Regionen und Zielgruppen erscheint daher sinnvoll und kann für Patienten ein weiterer Schritt hin zu einer sichereren Versorgung sein.

Interessenkonflikt: Die KVWL hat an den Handlungsempfehlungen mitgearbeitet. Frau M. Buchmann ist dort angestellt. Die anderen Autoren geben an, dass kein Interessenkonflikt besteht.

\section{Literatur}

1 World Health Organization. Safe Surgery Saves Lives Brochure. The Second Global Patient Safety Challenge 2008

2 Haynes $A B$, Weiser TG, Berry WR et al. A surgical safety checklist to reduce morbidity and mortality in a global population. New England Journal of Medicine 2009; 360: 491-499 
3 Shojania KG, Duncan BW, McDonald KM et al. Making health care safer: a critical analysis of patient safety practices. Rockville, MD: Agency for Healthcare Research and Quality; 2001

4 Aktionsbündnis Patientensicherheit. Handlungsempfehlungen zur Vermeidung von Eingriffsverwechslungen in der Chirurgie. Berlin; 2006

5 Aktionsbündnis Patientensicherheit. Handlungsempfehlungen zur sicheren Patientenidentifikation. Berlin; 2008

6 Aktionsbündnis Patientensicherheit. Leitfaden für APS-Arbeitsgruppen zur Erstellung und Verarbeitung von Handlungsempfehlungen. Berlin; 2014

7 Kassenärztliche Vereinigung Westfalen-Lippe. Handlungsempfehlung - Vermeidung von Eingriffsverwechselungen beim ambulanten Operieren. Dortmund 2013

8 Kassenärztliche Vereinigung Westfalen-Lippe. Handlungsempfehlung Sichere Patientenidentifikation in der ambulanten ärztlichen Versorgung. Dortmund 2013

9 Levesque DA, Prochaska JM, Prochaska JO et al. Organizational stages and processes of change for continuous quality improvement in health care. Consulting Psychology Journal: Practice and Research 2001; 53 : 139-153

10 Prochaska JM, Prochaska JO, Levesque DA. A transtheoretical approach to changing organizations. Administration and Policy in Mental Health and Mental Health Services Research 2001; 28: 247-261
11 Briner M, Kessler O, Pfeiffer $Y$ et al. Erste Schweizer Erhebung zum klinischen Risikomanagement im Spital. Zusammenfassung der Ergebnisse. Schweiz Ärztezeitung 2009; 90: 635-638

12 Rothmund M, Kohlmann T, Heidecke C-D et al. Einführung und Beurteilung von Maßnahmen zur Fehlerprävention in chirurgischen Kliniken: Ergebnisse einer aktuellen online-Befragung. (in press). Zeitschrift für Evidenz, Fortbildung und Qualität im Gesundheitswesen (ZEFQ) 2015

13 Renner D, Fishman L, Lessing C. Das Verwechslungsrisiko bei Eingriffen verringern. Dtsch Arztebl 2012; 109: 1016-1018

14 Muche-Borowski C, Nothacker M, Kopp I. Leitlinienimplementierung. Wie schließen wir die Lücke zwischen Evidenz und Anwender? Bundesgesundheitsblatt Gesundheitsforschung Gesundheitsschutz 2015; 58: $32-37$

15 Santos S, Lessing C, Schrappe $M$ et al. Entwicklung, Anwendung und Evaluation eines Konzeptes zur sicheren Patientenidentifikation im Krankenhaus - Wie kann der Wissenstransfer gelingen? (eFirst). Das Gesundheitswesen

16 Wachter RM. Fokus Patientensicherheit: Fehler vermeiden, Risiken managen. Berlin: ABW. Wissenschaftsverlag; 2010 